\title{
MODELO ADMINISTRATIVOY DE \\ EDUCACIÓN FINANCIERA PARA LAS \\ UNIDADES ECONÓMICAS EN PASQUILLA \\ (CUNDINAMARCA)
}

Leidy Marcela Murillo Ramírez

Cesar José Regino González

Linda Julieth Bernal Méndez Jenny Paola Torres Guillen

María Alejandra Cardona Suárez

James Osorio Muñoz'

Corporación Universitaria Minuto de Dios

\section{Resumen:}

Este proyecto de investigación se centra en la comunidad rural de Pasquilla, a través de un modelo de capacitación administrativa y formación en educación financiera, que brindará herramientas que permitan optimizar el manejo de los recursos, por lo que contribuirá con el mejoramiento de su calidad de vida. Así mismo, se enfoca en generar conciencia sobre la importancia de ser campesino y diversificar sus producciones haciéndolos competitivos en el mercado agropecuario. El proyecto de investigación se basa en métodos cualitativos y cuantitativos. Lo cualitativo se origina tras la observación y análisis de la visita a la zona y socialización con los habitantes.

Se pretende contribuir en el emprendimiento y asociación campesina, no tanto por sus logros económicos y financieros; sino por sus procesos de aprendizaje, gestión y vínculos sociales reflexivos que les permitirán obtener recursos naturales, de capacitación y una identidad como campesinos

Palabras clave: modelo; unidades; formalización, recursos; educación. 


\section{Abstract:}

This investigation is very important for this Pasquilla, in Ciudad Bolívar, Bogotá. This, through a guide of administrative training and financial education that would provide knowledge that can optimize resource management; it will contribute to improving your quality of life. For this reason, the objective is to expose about the importance of being a peasant and vary their productions making them competitive in the agricultural market. The project of investigations is focus about qualitative and quantitative methods. For example, of qualitative originates after the observation test and socialize with people in this zone.

The intention is to improve to leadership and peasant's association not so much for this economic achievements but for their education success and social ties that get natural resources of training and an identity as peasant.

Keywords: model; units; formalization; resources; education.

\section{Introducción}

Desde nuestra experiencia en la zona de Pasquilla evidenciamos que la mayoría de los habitantes dependen de su trabajo para ganarse la vida, sin embargo, las oportunidades de empleo en la zona rural suelen ser precarias y estar mal remuneradas. Por lo anterior, pretendemos impartir información con el fin de generar valor a sus actividades y diversificar sus unidades productivas; que contribuya a que los campesinos de la zona sean más activos en las tareas agropecuarias y minimizar la informalidad de las labores rurales que afectan los recursos de la vereda.

\section{Marco teórico}

Según el Comité de Seguridad Alimentaria Mundial (2015), los pequeños productores desempeñan un papel esencial para afianzar la seguridad alimentaria y la nutrición. Estos actores contribuyen a proporcionar una gran variedad de beneficios adicionales, a la creación de empleo en las zonas rurales, 
la reducción de la pobreza y el fortalecimiento de la ordenación sostenible de los recursos naturales. No obstante, a menudo ellos mismos se ven afectados por la inseguridad alimentaria y la malnutrición, pues alrededor del $80 \%$ de las personas pobres y que padecen inseguridad alimentaria viven en las zonas rurales y la mayoría de los campesinos pobres son pequeños productores familiares de alimentos (Rodríguez-Parra, Rodríguez Pardo y Bernal, 2019).

De acuerdo con el mismo comité, en junio de 2015, se celebró un foro de alto nivel sobre la vinculación de los pequeños productores con los mercados en el que se examinaron las implicaciones para las políticas, los retos y las enseñanzas extraídas de ejemplos concretos de agricultores que habían aprovechado las oportunidades para establecer vínculos sostenibles con los mercados.

Bill Vorley (2012), explica que el aumento de la informalidad constituye un desafío para el desarrollo sostenible de largo plazo. Aunque los mercados informales ofrecen ventajas fundamentales como el acceso y la flexibilidad, y representan espacios de creatividad y confianza entre los pequeños agricultores y otros actores que participan en el mercado, también tienen un lado oscuro que comprende cosas como la poca transparencia y la inocuidad alimentaria, que son antecedentes pobres en cuanto al impacto ambiental y la seguridad laboral, y a veces corrupción, criminalidad, monopolios y cárteles.

Algunos investigadores advierten el riesgo de que las familias pobres queden atrapadas en la pobreza, incluso cuando la informalidad puede ofrecer soluciones en el corto plazo. Así mismo, estudios recientes insinúan que en crisis y/o durante una reducción de la actividad económica global, el sector informal puede ser golpeado de forma más fuerte que el sector formal, contrariamente a la interpretación popular de que el sector informal constituye un mejor refugio en tiempos de crisis económica. 
De acuerdo con Carla Mucavi (2018), la Organización de las Naciones Unidas para la Agricultura y la Alimentación (FAO) ha expresado su satisfacción por una histórica resolución de la Asamblea General de las Naciones Unidas que adopta la "Declaración sobre los derechos de los campesinos y otras personas que trabajan en las zonas rurales", que la Asamblea General aprobó el 17 de diciembre de 2018. Dicha declaración, tiene como objetivo proteger los derechos de todas las poblaciones rurales, incluidos los campesinos, los trabajadores agrícolas y rurales y los pueblos indígenas; al tiempo que reconoce su contribución al desarrollo sostenible y la biodiversidad y los desafíos a lo que se enfrentan.

Aseguró Mucavi que "la FAO aplaude la adopción de la declaración. A la población rural se le ha dejado atrás de forma sistemática: constituye la gran mayoría de los pobres del mundo, generalmente tienen salarios más bajos y menos acceso al agua, energía, protección social y otros servicios que son esenciales para su desarrollo sostenible. Esta es una oportunidad para cambiar esta realidad", quien se desempeña como directora de la Oficina de Enlace de la FAO para las Naciones Unidas en Nueva York.

Los derechos específicos reconocidos por la declaración incluyen el derecho a una alimentación adecuada, a la tierray al agua. La declaración sostiene también la necesidad de respetar la identidad cultural y el conocimiento tradicional de la población rural; así, como la exigencia de proporcionar protección social y garantizar la igualdad de género en las zonas rurales.

La adopción de la declaración es la culminación de un proceso negociador inclusivo que se prolongó durante seis años, liderado por Bolivia. La FAO ha apoyado este proceso para la adopción de la declaración, que está vinculada a la mayoría de los principios y directrices adoptados por el Comité de Seguridad Alimentaria Mundial y varios órganos de la misma organización. 
En particular, la declaración hace referencia a las directrices voluntarias sobre la gobernanza responsable de la tenencia de la tierra, la pesca y los bosques en el contexto de la seguridad alimentaria nacional y otros acuerdos a nivel mundial como el "Tratado Internacional sobre los Recursos Fitogenéticos para la Alimentación y la Agricultura". Los temas y valores que sustentan la declaración se relacionan además con otros aspectos del trabajo de la FAO, incluyendo los pueblos indígenas.

Para el caso de Colombia, explica Posada (2017), cuenta con una ruralidad muy diversa, la cual ha sido inadecuadamente atendida por las políticas públicas durante los últimos gobiernos. Esta realidad ha provocado condiciones que van en desmedro de la economía campesina, reflejada en situaciones como alta concentración de la tierra, desmonte de los sistemas de asistencia técnica e investigación, subsidios tendientes a beneficiar a grandes capitalistas rurales, altos precios de insumos comparado con los países vecinos, escasas oportunidades de una pertinente educación, difícil acceso a sistemas de salud, exigua posibilidad de acceder a una pensión de vejez, entre muchas otras. Lo anterior, ha generado procesos de aislamiento y precariedad evidentes en muchas zonas rurales de Colombia.

En relación con los procesos productivos de la economía campesina, el uso de su recurso productivo más importante, la mano de obra familiar, ha sido una característica primordial como estrategia para superar la falta de acceso a tierra y capital. Igualmente, es utilizado como maniobra para superar los efectos negativos derivados de la implementación de las políticas públicas.

La discusión relacionada con economía campesina, su significado e importancia para Latinoamérica ha sido muy nutrida. Un primer acercamiento a esta discusión es aquella que plantea la racionalidad productiva de las familias campesinas en relación con sus condiciones y situación inmediata de producción, de organización, gestión y planificación de actividades. Es caracterizada por el uso de la fuerza de trabajo familiar, así como escasos recursos de tierra y tecnología. 


\section{Resultados}

- Los resultados de la investigación reflejan que las comunidades rurales deben implementar procesos productivos a través de prácticas formales que permitan mejorar su calidad de vida.

- El emprendimiento y asociación campesina, no tanto por sus logros económicos, financieros y jurídicos; sino por sus procesos de aprendizaje, gestión y vínculos sociales reflexivos les ha permitido obtener recursos naturales, físicos, financieros y de capacitación.

\section{Impacto Positivo}

- Contribuir en el emprendimiento y la asociación campesina, por medio de la formalización.

- Incentivar las buenas prácticas agrícolas las cuales permitan mejorar la producción y conservación de los recursos naturales.

- Impactar positivamente en las finanzas de los campesinos, mejorando así su calidad de vida.

\section{Conclusiones}

- Se pretende contribuir en el emprendimiento de la zona rural de Pasquilla, además buenos hábitos financieros, a través de la capacitación por medio de las cartillas educativas.

- Mayores posibilidades de posicionarse en el mercado, pueden asociarse con otras personas o con otras empresas para lograr una mayor competitividad.

- Promover una actividad económica permanente en el área rural, por lo cual se produciría un fuerte cambio en la actividad productiva. 


\section{Referencias bibliográficas}

Bill Vorley, E. d. (2012). rimisp.org. Recuperado el 31 de Agosto de 2019, de http://www.rimisp.org/wpContent/ files__ mf/1377793224agricultoresdepequenaescalaenelmercadoglobalizadotomandodecisionesenunmundocambiante.pdf

Comité de Seguridad Alimentaria mundial. (2015). fao.org. Recuperado el 31 de agosto de 2019, de http://www.fao.org/ cfs/cfs-home/activities/smallholders/es/

Mucavi, C. (2018). Organizacion de las naciones unidas para la alimentacion y la agricultura. Recuperado el 31 de Agosto de 2019, de http://www.fao.org/news/story/es/ item/1175226/icode/
Posada, J. C. (2017). Identificación de ventajas y desventajas de los canales de comercialización en las economías campesinas de dos municipios de Meta y Cundinamarca, Colombia. Revista de investigacion, desarrollo e innovacion 8(1). 35 47. https://doi.org/10.19053/20278306. v8.n1.2017.7369

Rodríguez-Parra, P., Rodríguez Pardo, D. y Bernal, M. (2019). Reingeniería social para la promoción de la equidad y la prosperidad en las comunidades menos favorecidas. Bogotá: Editorial Fundación Universitaria San Mateo. Recuperado de https://palma. sanmateo.edu.co/ 